\title{
Hepatitis B virus Pres2-mutant large surface antigen activates store-operated calcium entry and promotes chromosome instability
}

\author{
Tim Ting-Chung Yen ${ }^{1}$, Anderson Yang ${ }^{1}$, Wen-Tai Chiu ${ }^{2,3}$, Tian-Neng Li $^{1}$, Lyu-Han \\ Wang $^{1}$, Yi-Hsuan Wu ${ }^{1}$, Hui-Chen Wang ${ }^{4}$, Linyi Chen ${ }^{5,6}$, Wen-Ching Wang ${ }^{1}$, Wenya \\ Huang ${ }^{7}$, Chien-Wen Chang ${ }^{8}$, Margaret Dah-Tsyr Chang ${ }^{1,6}$, Meng-Ru Shen ${ }^{3,9}$, Ih-Jen \\ Su ${ }^{3,10,11}$, Lily Hui-Ching Wang ${ }^{1,6}$ \\ ${ }^{1}$ Institute of Molecular and Cellular Biology, National Tsing Hua University, Hsinchu 300, Taiwan \\ ${ }^{2}$ Department of Biomedical Engineering, National Cheng Kung University, Tainan 701, Taiwan \\ ${ }^{3}$ Center of Infectious Diseases and Signal Transduction, National Cheng Kung University, Tainan 701, Taiwan \\ ${ }^{4}$ Institute of Pharmaceutics, Development Center for Biotechnology, Taipei 22180, Taiwan \\ ${ }^{5}$ Institute of Molecular Medicine, National Tsing Hua University, Hsinchu 300, Taiwan \\ ${ }^{6}$ Department of Medical Science, National Tsing Hua University, Hsinchu 300, Taiwan \\ ${ }^{7}$ Department of Medical Laboratory Science and Biotechnology, National Cheng Kung University, Tainan 701, Taiwan \\ ${ }^{8}$ Department of Biomedical Engineering and Environmental Sciences, National Tsing Hua University, Hsinchu 300, Taiwan \\ 'Department of Pharmacology, National Cheng Kung University, Tainan 701, Taiwan \\ ${ }^{10}$ National Institute of Infectious Diseases and Vaccinology, National Health Research Institutes, Tainan 704, Taiwan \\ ${ }^{11}$ Department of Biotechnology, Southern Taiwan University of Science and Technology, Tainan 710, Taiwan \\ Correspondence to: Lily Hui-Ching Wang, e-mail: lilywang@life.nthu.edu.tw \\ Ih-Jen Su, e-mail: suihjen0704@stust.edu.tw
}

Keywords: SOCE, ER stress, hepatitis B virus, ground-glass hepatocytes, aneuploidy

Received: August 21, 2015

Accepted: February 28, 2016

Published: March 16, 2016

\section{ABSTRACT}

Hepatitis B virus (HBV) is a driver of hepatocellular carcinoma, and two viral products, $X$ and large surface antigen (LHBS), are viral oncoproteins. During chronic viral infection, immune-escape mutants on the preS2 region of LHBS (preS2-LHBS) are gain-of-function mutations that are linked to preneoplastic ground glass hepatocytes (GGHs) and early disease onset of hepatocellular carcinoma. Here, we show that pres2LHBS provoked calcium release from the endoplasmic reticulum (ER) and triggered stored-operated calcium entry (SOCE). The activation of SOCE increased ER and plasma membrane (PM) connections, which was linked by ER-resident stromal interaction molecule-1 (STIM1) protein and PM-resident calcium release-activated calcium modulator 1 (Orai1). Persistent activation of SOCE induced centrosome overduplication, aberrant multipolar division, chromosome aneuploidy, anchorage-independent growth, and xenograft tumorigenesis in hepatocytes expressing preS2-LHBS. Chemical inhibitions of SOCE machinery and silencing of STIM1 significantly reduced centrosome numbers, multipolar division, and xenograft tumorigenesis induced by preS2-LHBS. These results provide the first mechanistic link between calcium homeostasis and chromosome instability in hepatocytes carrying preS2-LHBS. Therefore, persistent activation of SOCE represents a novel pathological mechanism in HBV-mediated hepatocarcinogenesis.

\section{INTRODUCTION}

Hepatitis B virus (HBV) infection causes fulminant hepatitis and advanced liver disease. In endemic areas, most chronic liver diseases, including cirrhosis and hepatocellular carcinoma (HCC), are associated with
HBV [1-3]. Two HBV-encoded oncoproteins have been identified, including $X$ and large surface antigen (LHBS). The $\mathrm{X}$ protein functions as a robust gene transactivator and a binding partner of p53 [4]. In contrast, the role of LHBS in the development of HCC is not clear. 
During chronic HBV infection, viral surface antigen (HBsAg) is the most abundant viral product detected in the liver and circulation in patients. The surface antigen gene contains three segments (preS1, preS2, and S) with two internal translation start sites that produce three polypeptides with a common C-terminus: large (LHBS), middle (MHBS), and small surface antigens (HBS). Clinical assessment of HBsAg usually uses antibodies specific for the common $\mathrm{S}$ region to detect all three types of HBsAg. In early stages of chronic HBV infection, HBsAg exhibits diffused cytoplasmic distribution in the liver (Figure 1A). Occasionally, hepatocytes expressing inclusion-like HBsAg, known as type I ground glass hepatocytes (GGHs), can be found. In advanced disease, histological preneoplastic changes are detected in clusters of hepatocytes with a specific marginal distribution of HBsAg, known as type II GGHs or marginal type GGHs (Figure 1A) [5]. Notably, the development of different GGHs reflects the emergence of immune-escape mutants during chronic viral infection [6-9]. In addition, approximately $45 \%$ to $60 \%$ of HCC patients had preS mutants, suggesting these mutations are gain-of-function mutations during hepatocarcinogenesis $[6,10,11]$. In-frame truncations of the immune-dominant preS2 region with or without a preS2 start codon mutation were isolated from type II GGHs using laser capture microdissection and in the serum of patients with advanced liver diseases and HCC [7-9]. Strikingly, viral preS2 mutants were found in nearly half of children with $\mathrm{HCC}$, in contrast to its absence in children with chronic HBV infection [11]. In another retrospective study of 42 children with $\mathrm{HCC}$, preS deletion mutants were detected in over $90 \%$ of cases positive for HBV, among which $74 \%$ of cases contained preS2 deletion mutants [12]. Notably, the preS2 deletion consistently appeared with the deletion of nucleotides $4-57$, corresponding to a loss of 18 amino acids, including one CD8 T cell epitope, in the livers or circulation $[7,9,12,13]$. These studies implied that preS2 truncation mutants are immune-escape gain-of-function mutations that contribute to the development of HCC during chronic HBV infection.

The oncogenic characteristics of preS2 mutants have been investigated recently. Transgenic mice expressing the most typical preS2 mutant (nucleotides 4-57 deletion) LHBS protein developed liver dysplastic changes and ultimately $\mathrm{HCC}$, indicating that preS2LHBS is a promising viral oncoprotein $[14,15]$. The retention of preS2-LHBS in the endoplasmic reticulum (ER) induced ER stress and thereby initiated the ER stress-dependent VEGF/Akt/mTOR and NF- $\mathrm{kB} / \mathrm{COX} 2$ signaling pathways, as well as oxidative DNA damage and centrosome overduplication $[16,17]$. Independent of the ER stress pathways, preS2-LHBS directly interacted with c-Jun activation domain-binding protein 1 (JAB1), which led to the degradation of $\mathrm{p} 27$ and thereby promoted the hyperphosphorylation of retinoblastoma [18].
In addition, preS2-LHBS directly interacted with importin $\alpha 1$, thereby blocked the nuclear transport of an essential DNA repair and recombinant factor, Nijmegen breakage syndrome 1 (NBS1), upon DNA damage, and subsequently impeded DNA double-stranded break repair [19]. Notably, transgenic mice carrying preS2-LHBS displayed higher genomic instability than transgenic mice carrying HBV X protein using array-based comparative genomic hybridization. Consistently, human type II GGHs carrying preS2 mutants also exhibited an increased level of DNA double-strand breaks [19]. These results indicate that HBV preS2 mutants may facilitate genomic instability in hepatocytes during disease progression. Notably, genomic instabilities were detected not only in individual genes but also at the chromosome level in these transgenic mice [19]. Similarly, the genetic compositions in $\mathrm{HBV}$-associated $\mathrm{HCC}$ are complicated with changes in individual chromosomes, such as gains of chromosomes $1 \mathrm{q} / 6 \mathrm{p} / 8 \mathrm{q}$ and losses of chromosomes 4q, 8p, 16q, and others [20]. Although the induction of oxidative DNA damage and the inhibition of DNA repair could provide an explanation for the changes at the gene level, whether and how HBV promotes chromosome number instability during disease progression were not investigated.

In the present study, we aimed to explore whether the subcellular distribution of preS2-LHBS is essential for its oncogenic properties during disease progression. Unlike conventional ER-resident proteins that display typical perinuclear distribution in the cell, preS2-LHBS was mostly detected at the cell margin in type II GGHs and HuH-7 cells following transient transfection [9]. Marginal distribution of preS2-LHBS in GGHs prompted us to investigate whether this particular subcellular localization is involved in the ER-plasma membrane (PM) connection under stress conditions. In the present study, we show that the expression of preS2-LHBS increased ER and plasma membrane (PM) connections through the activation of store-operated calcium entry (SOCE), which is mediated by the interaction between stromal interaction molecule-1 (STIM1) and a calcium release-activated calcium modulator 1 (Orai1). The activation of SOCE not only increased the intracellular calcium concentration but also provoked centrosome overduplication. By time-lapse imaging, hepatocytes carrying preS2-LHBS underwent aberrant multipolar division and ultimately reached chromosome aneuploidy. Finally, we show that preS2LHBS is capable of inducing xenograft tumorigenesis, and this effect was largely suppressed by the depletion of STIM1. Thus, we suggest that the activation of SOCE machinery is involved in chromosome instability in the development of HBV-mediated HCC. 


\section{RESULTS}

\section{PreS2-LHBS promotes ER-PM connections through ER stress}

In contrast to the diffuse cytoplasmic distribution of wild type (WT)-LHBS, preS2-LHBS was usually detected at the cell margin of GGHs in the liver (Figure 1A). Similarly, WT-LHBS and preS2-LHBS displayed diffuse and marginal localization, respectively, in the hepatic progenitor line NeHepLxHT [21] (Figure 1B). As preS2LHBS is an ER-resident protein, we asked whether the cytoplasmic distribution of bulk ER is affected in these cells. To this end, cells were infected with recombinant baculoviruses for exogenous expression of red fluorescent protein (RFP) fused with the myristoylation/palmitoylation sequence from Lck tyrosine kinase (as PM-RFP) and green fluorescence protein (GFP) fused with the ER signal sequence of calreticulin and KDEL (as ER-GFP). By monitoring the spatial correlation between ER-GFP and PM-RFP, we found that overlapping signals at the cell margin were increased in the presence of WT- and preS2-LHBS (Figure 1C). Quantitatively, the overlapping peak signals associated with ER-GFP and PM-RFP were detected in approximately $30 \%$ of the WT-LHBS cells and $75 \%$ of the preS2-LHBS cells. Less than $5 \%$ of the control cells displayed marginal ER-GFP (Figure 1D). Coordinately, greater than $60 \%$ of the preS2-LHBS cells displayed peripheral recruitment of preS2-LHBS, as indicated by the peak fluorescence signal shown near PM-RFP (Figure 1E, 1F). Thus, we concluded that the ER-PM connections were increased in the presence of preS2-LHBS.

To investigate whether ER stress is involved in the ER-PM connection, we treated cells with thapsigargin, which inhibits SERCA and activates ER stress via the depletion of ER calcium storage. Peripheral localization of ER-GFP was significantly increased in control and WTLHBS cells with thapsigargin. In contrast, the marginal distribution of ER-GFP was not affected by thapsigargin in preS2-LHBS cells (Figure 2A, 2B). Given that ER stress is expected to increase calcium efflux from the ER, we confirmed that the preS2-LHBS cells displayed increased intracellular calcium levels compared with the control and WT-LHBS cells (Figure 2C). Together, these results indicated that preS2-LHBS promotes the ER-PM connection and disrupt calcium homeostasis through ER stress.

\section{SOCE provokes the marginal distribution of LHBS}

Store-operated calcium entry is a major calcium influx pathway controlling the intracellular calcium concentration in the cell [22]. To investigate SOCE activity, cells were pre-loaded with a fluorescent calcium sensor, treated with thapsigargin, and incubated in calcium-free medium to deplete all intracellular calcium. SOCE activity was then measured via the detection of calcium influx after thapsigargin was removed and replaced with calcium-containing medium. Compared with the control cells, a significant increase in total calcium influx was detected in the preS2-LHBS cells (Figure 3A). Given that SOCE-mediated calcium influx depends on the interaction between STIM1 and Orai1 [23], the formation of STIM1-Orai1 puncta was detected in preS2-LHBS cells. In WT-LHBS cells, the STIM-Orail puncta were only detected after thapsigargin treatment (Figure 3B). Next, we explored whether the marginal distribution of preS2-LHBS depended on the peripheral recruitment of STIM1. Notably, the depletion of STIM1 abolished the peripheral recruitment of preS2-LHBs (Figure 3C, 3D). These results indicated that the marginal distribution of preS2-LHBS is mediated through the recruitment of STIM1 toward PM-localized Orail following the activation of SOCE machinery.

\section{Persistent SOCE activation depends on calcium efflux from the $\mathrm{ER}$}

Type II GGHs with marginally localized LHBS were persistent in the liver in advanced stages of chronic HBV infection [5, 6, 24, 25]. These observations implied that ER-PM connections are maintained constantly in type II GGHs. Given that the STIM1-Orail interaction is critical for this ER-PM connection, we asked whether the restoration of ER calcium homeostasis may retract the ER-PM connection in preS2-LHBS cells. To reduce ER calcium leakage in these cells, we increased the cytoplasmic calcium by adding extra calcium in the culture medium (final calcium concentration of $5 \mathrm{mM}$ ). Strikingly, STIM1-Orail puncta in preS2-LHBS cells were lost after adding extra calcium (Figure 4). Further treatment with thapsigargin, which blocked the ER calcium influx, rescued the formation of STIM1-Orail puncta in preS2LHBS cells under a high calcium condition (Figure 4, upper panels). Similarly, we showed that thapsigargin induced STIM1-Orail puncta in WT-LHBs cells, which persisted in the high calcium condition (Figure 4, lower panels). Thus, insufficient calcium storage in the ER is likely the major driving force for the connection between STIM1 and Orai1. These results implied that persistent STIM1-Orail connections in preS2-LHBS-expressing cells depend on constant calcium efflux from the ER, as a result of persistent ER stress.

\section{SOCE promotes centrosome overduplication, multipolar division, and aneuploidy}

A potential outcome of constant SOCE activation is the activation of calcium-dependent calpain proteases because of elevated intracellular calcium. Notably, 
A

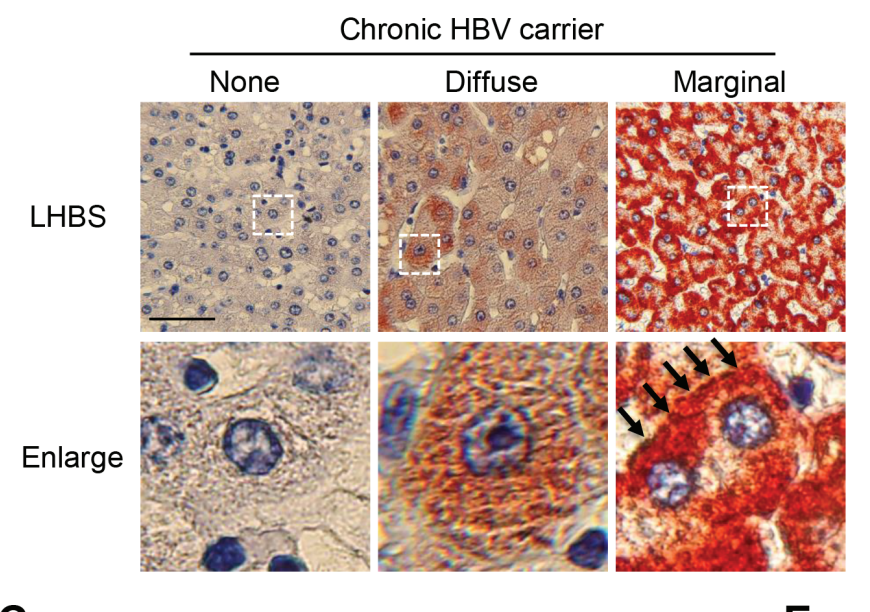

B

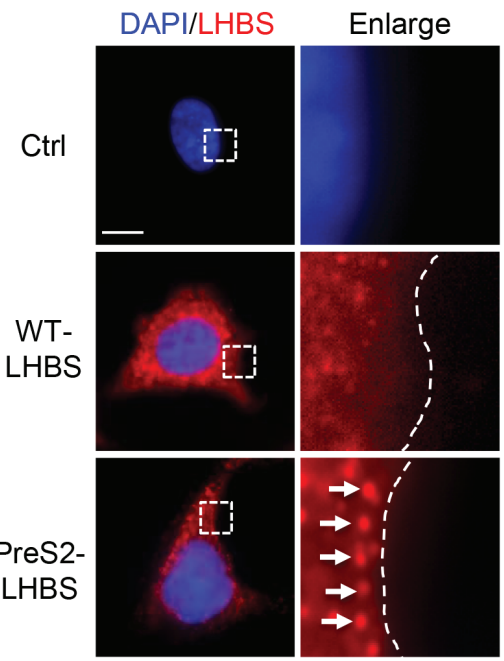

E
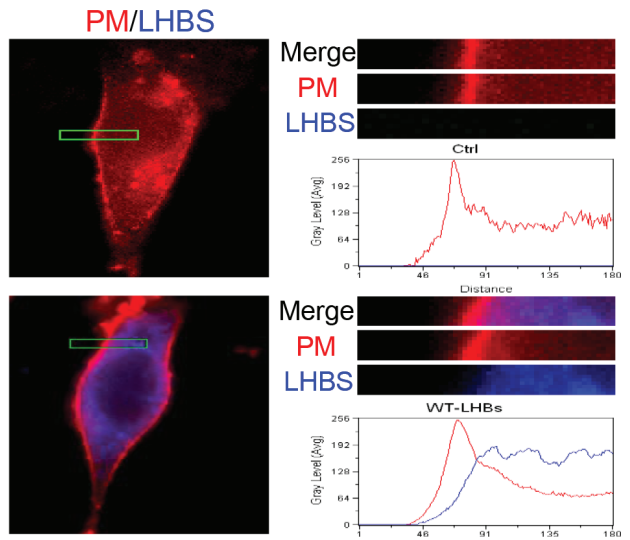

LHBS
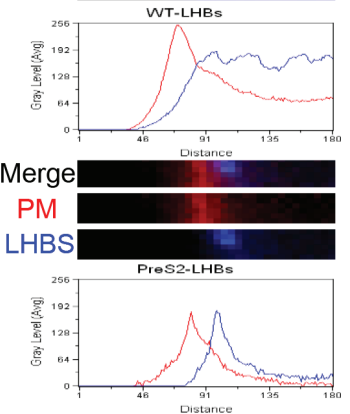

$\mathbf{F}$
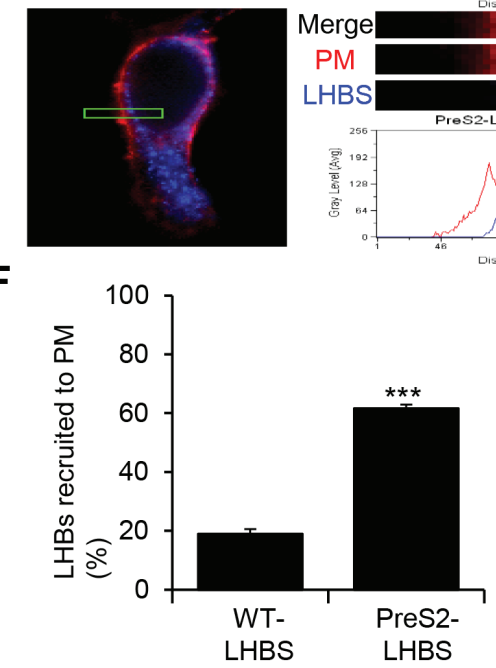

Figure 1: Intracellular distribution of the ER and LHBS. (A) Immunohistochemical staining for LHBS was performed on cirrhotic liver sections obtained from chronic HBV carriers. Representative images displaying hepatocytes with absent, diffuse, and marginal LHBS expression patterns. Black arrows indicate peripheral distribution of LHBS in type II GGHs. Scale bar, $100 \mu \mathrm{m}$. (B) NeHepLxHT cells that expressed a control (Ctrl) tag, WT-LHBS, or preS2-LHBS were immunostained for LHBS (red) and counterstained with DAPI (blue). Enlarged images of the boxed areas are shown. Dashed lines indicate the edges of the cells. White arrows indicate the peripheral distribution of preS2-LHBS. Scale bar, $10 \mu \mathrm{m}$. (C, E) Immunofluorescence images of intracellular distributions of PM (red), ER (green), and LHBS (blue) are shown. The upper right panels show enlarged images of the boxed area from the corresponding left images. Line scanning profiles show the grey level signal intensity of the indicated markers in the enlarged area. Scale bar, $10 \mu \mathrm{m}$. (D) Quantitative results show that a percentage of cells displayed a peak ER signal intensity that overlapped with the PM, which indicates the peripheral ER. (F) Percentages of cells that displayed marginal LHBS are shown. Marginal recruitment of LHBS was defined by the detection of the peak signal intensity of LHBS that partially overlapped with the PM profile, as shown in the preS2-LHBS cells $(\mathbf{E}) .{ }^{* * *} p<0.001$. 
A

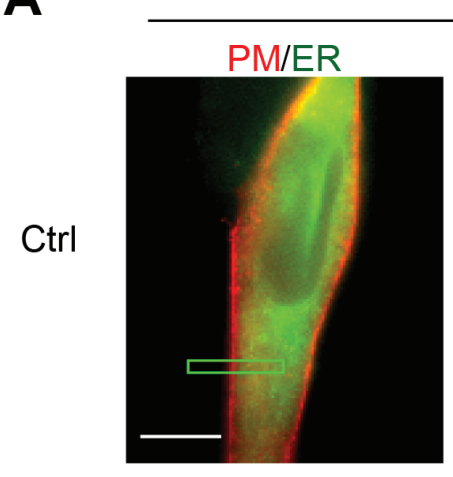

DMSO
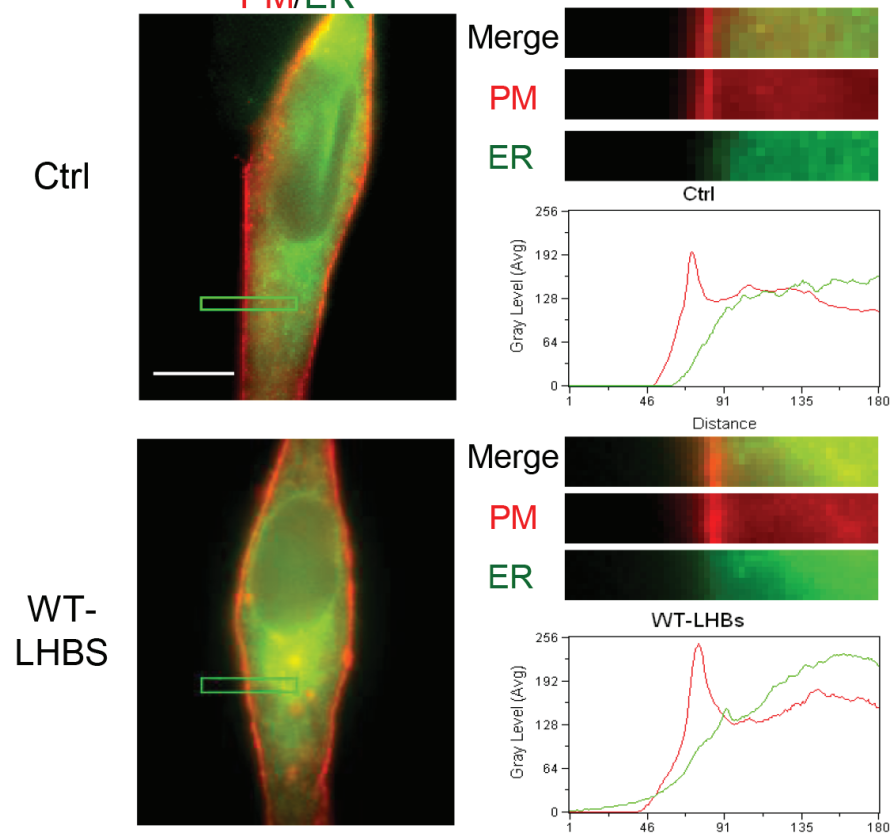

ER
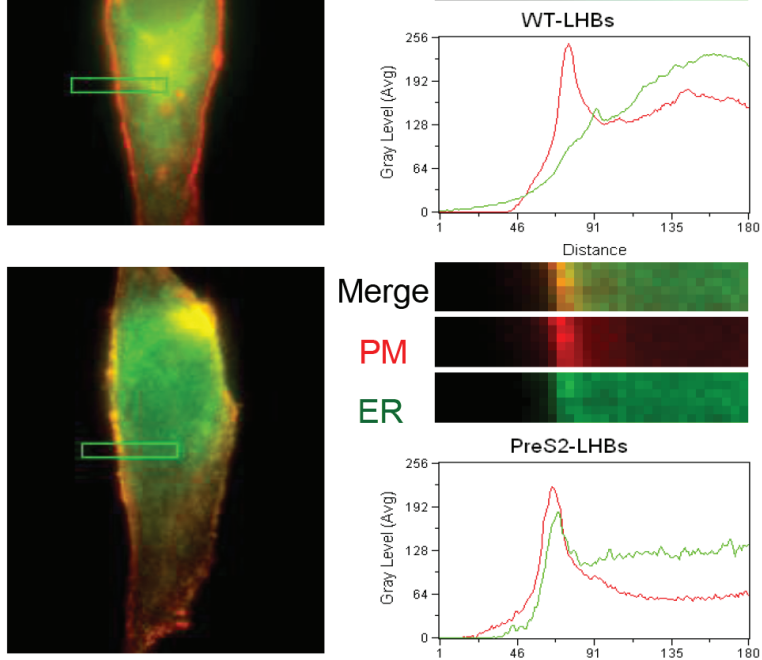

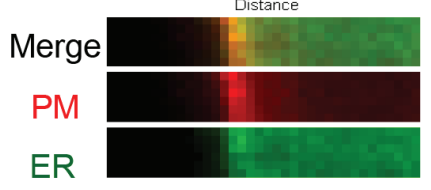

PreS2-LHBs

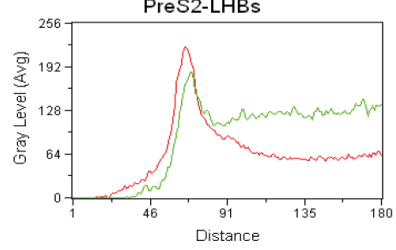

Thapsigargin
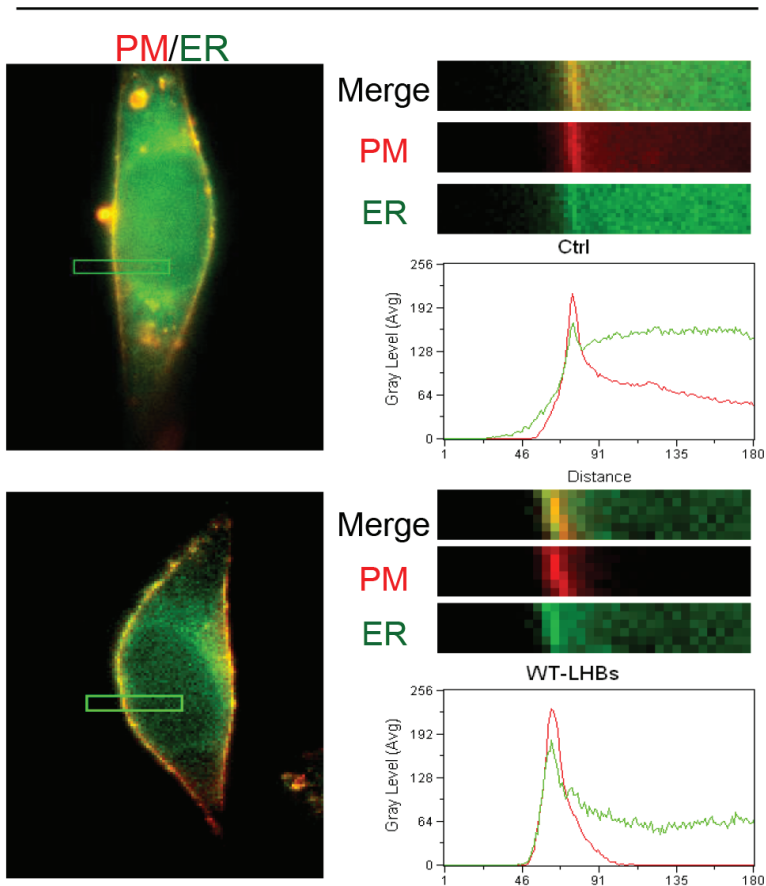

WT-LHBs
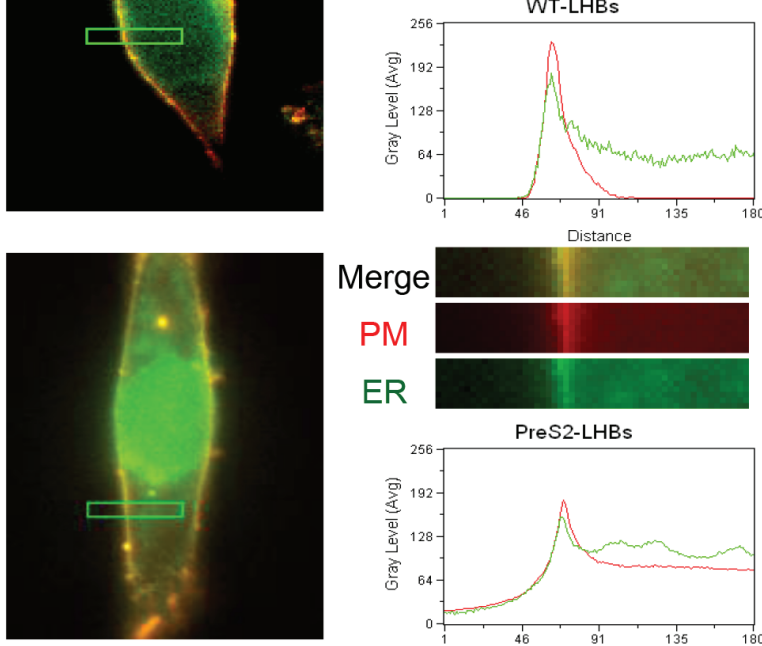

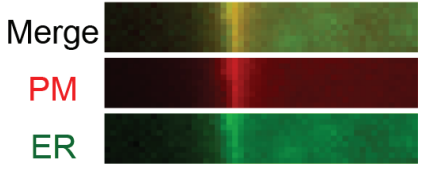

PreS2-LHBs

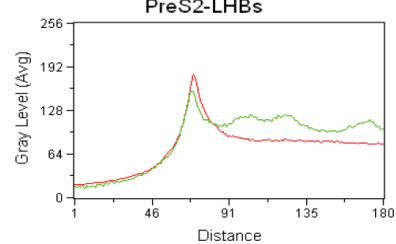

B
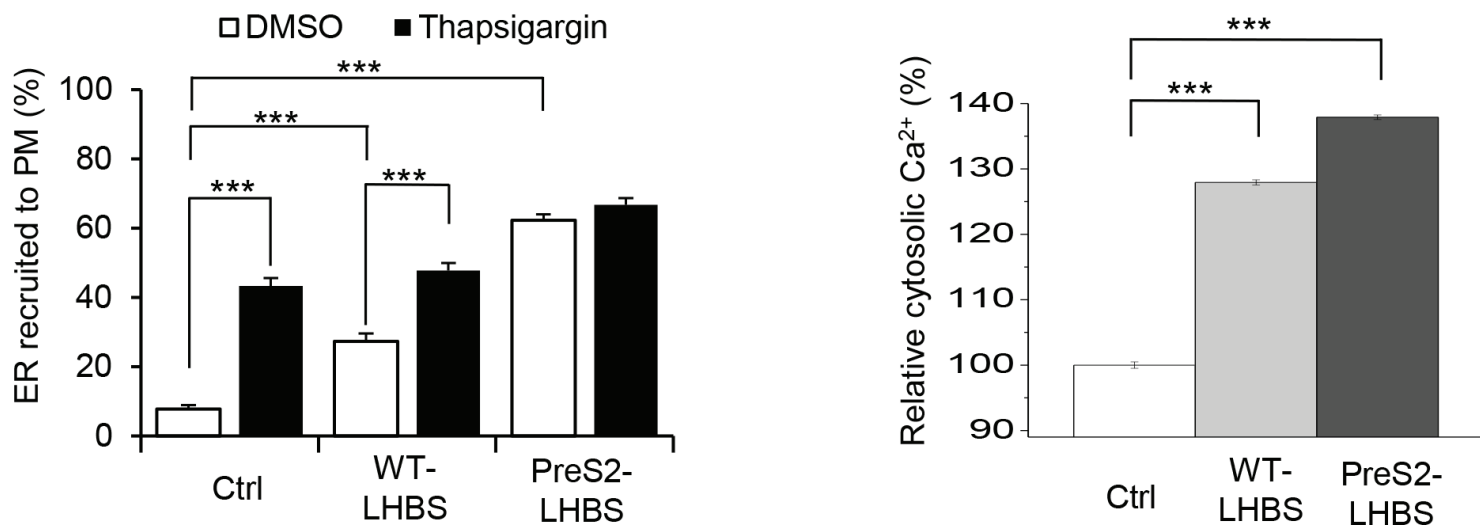

Figure 2: ER stress increases ER-PM connections. (A) Subcellular localization of PM (red) and ER (green) in indicated stable cell lines following the induction of ER stress. Cells were pre-treated with BacMam baculoviruses for the transduction of ER-GFP and PM-RFP for $48 \mathrm{~h}$, followed by a 15-min treatment with DMSO or thapsigargin. The upper right panels show enlarged images of the boxed area from the corresponding images. Line scanning profiles show the grey level signal intensity of ER (green line) and PM (red line) in the enlarged area. Scale bar, $10 \mu \mathrm{m}$. (B) Percentage of cells with ER recruitment toward the PM is defined by the detection of the ER peak signal intensity that overlapped with the PM peak profile. (C) The intracellular calcium concentration was measured by Fura-2 intensity. *** $p<0.001$. 
A

B
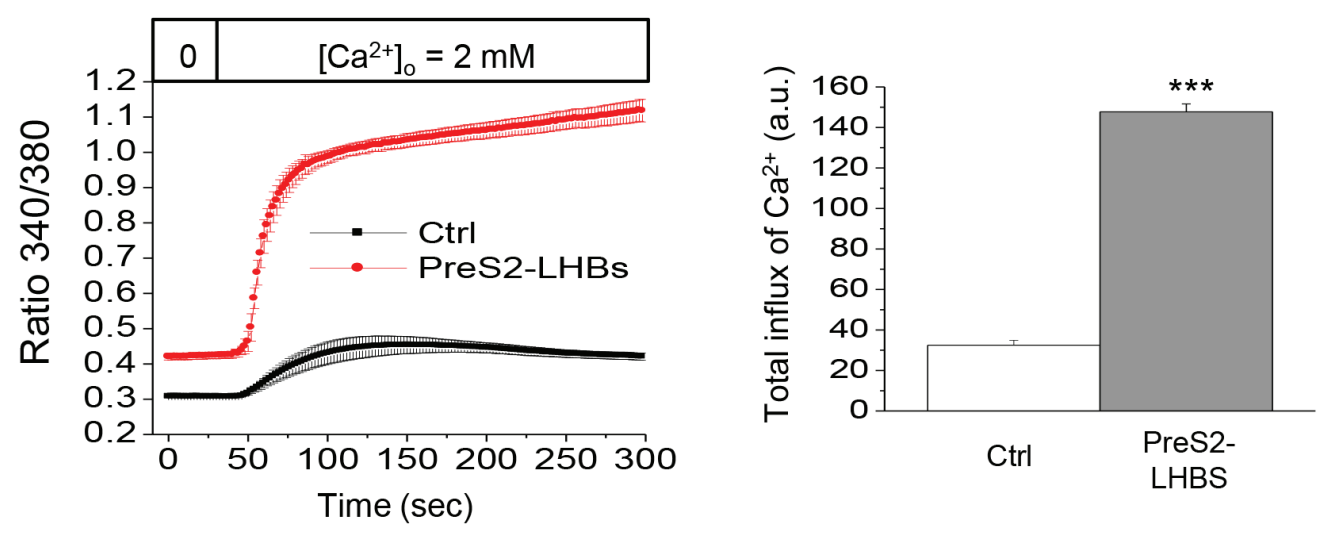

STIM1 Orai1 Merge

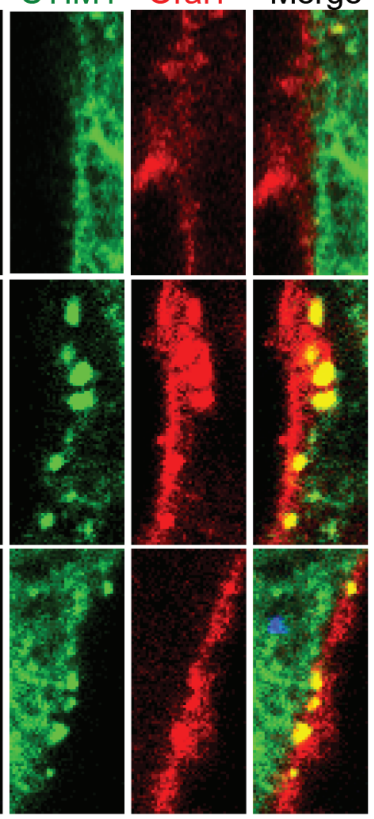

D

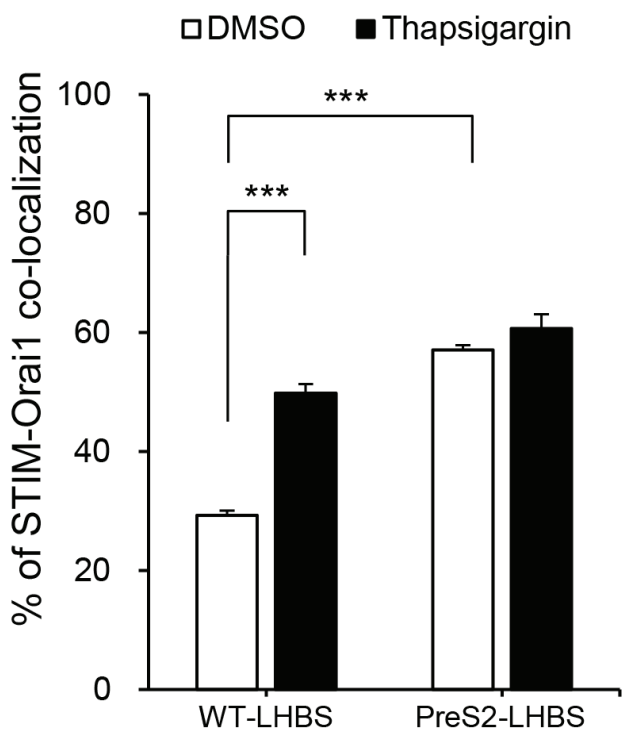

C

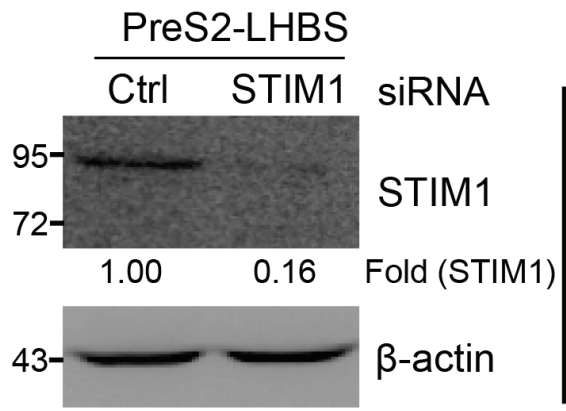

PreS2-LHBS / PM / DAPI

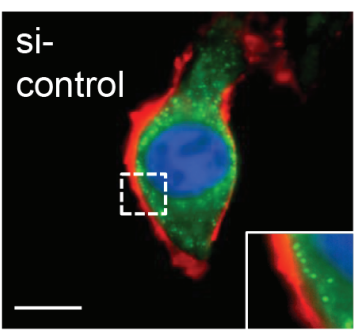

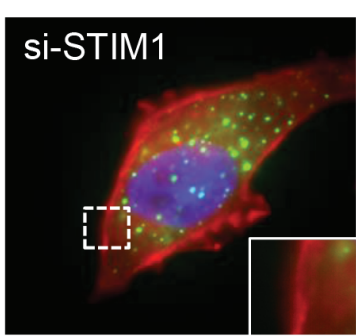

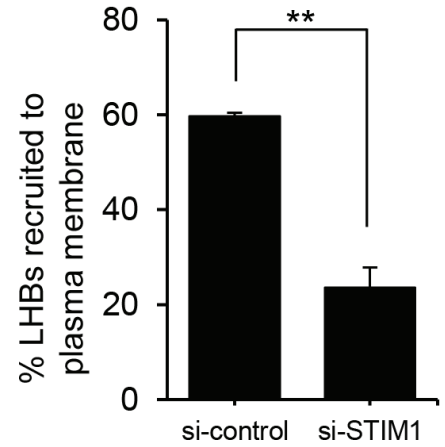

Figure 3: SOCE activation and peripheral recruitment of STIM1 in hepatocytes under ER stress. (A) SOCE activity was examined in control and preS2-LHBS cells by measuring calcium influx over time after being released from calcium-free medium and thapsigargin. The quantitative results obtained from at least 50 cells are displayed in the right panel (a.u., arbitrary units). (B) STIM1 (green) and Orail (red) formed puncta at the cell margin in preS2-LHBS cells. In WT-LHBS cells, STIM1-Orai1 puncta were only detected after thapsigargin treatment. The percentage of cells that contained overlapping STIM1-Orail puncta at the cell periphery is shown in the right panel. Scale bar, $10 \mu \mathrm{m}$. (C) STIM1 depletion in preS2-LHBS cells was confirmed by Western blotting. The numbers below the blot indicate the STIM1 fold reduction. (D) Depletion of STIM1 abolished the recruitment of preS2-LHBS toward the cell margin. Quantitative results are presented in the right panel. $* * p<0.01 ; * * * p<0.001$. 


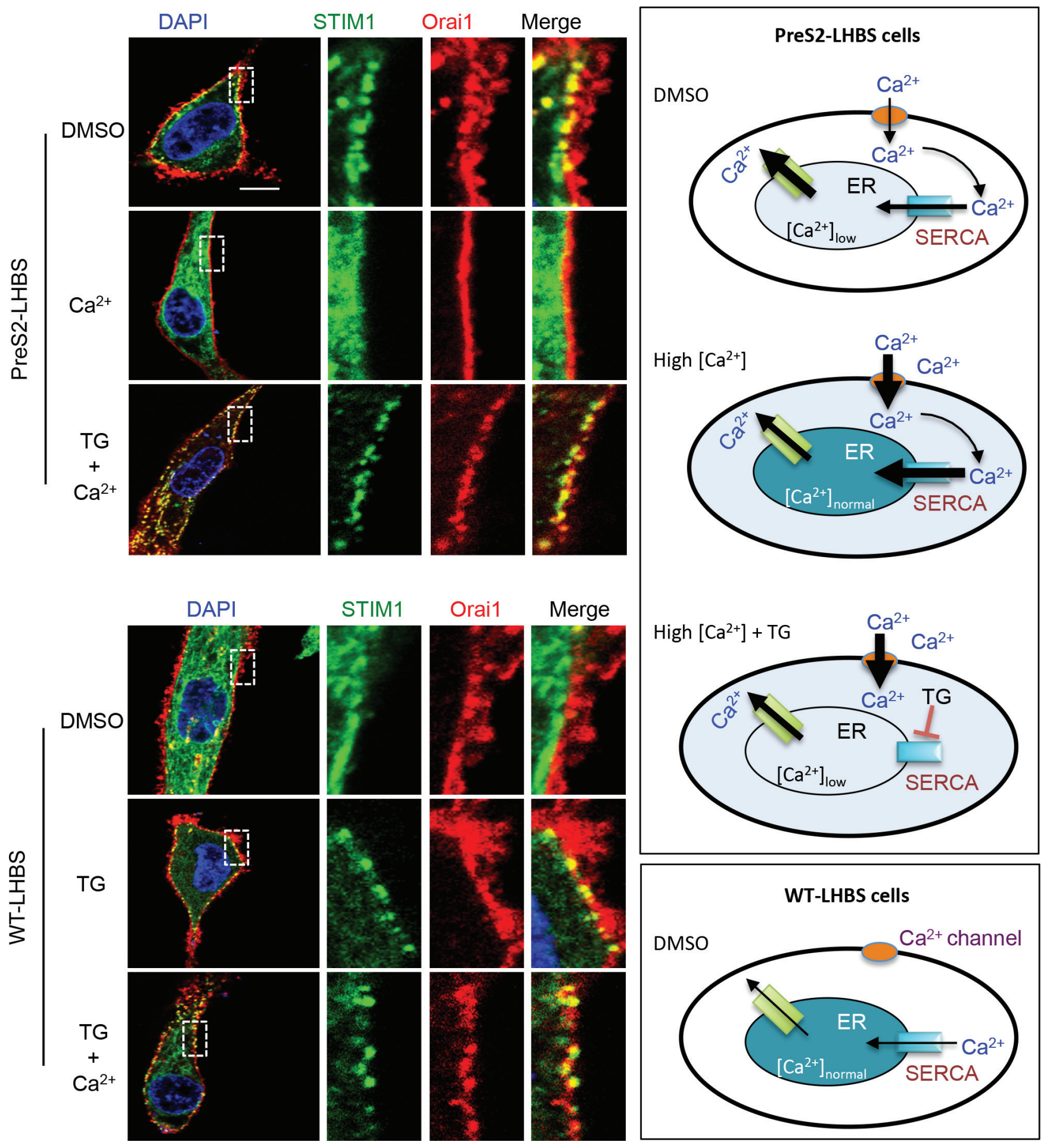

Figure 4: Activation of SOCE depends on the calcium concentration in the ER. EGFP-STIM1 (green) and mOrange-Orai1 (red) were transiently expressed in WT-LHBS and preS2-LHBS following treatment with DMSO, thapsigargin (TG), and/or an additional $4 \mathrm{mM} \mathrm{Ca}^{2+}$ in culture media. Representative images are shown on the left. Right panels illustrate the relative concentration of cytosolic and ER calcium under various conditions. In preS2-LHBS cells, ER stress promotes calcium release from the ER and thereby induces the STIM1-Orail connection and SOCE activation. Replenishment of ER calcium via an increase in the calcium concentration in the culture medium abolished the STIM1 and Orail connection in preS2-LHBS cells. In contrast, co-treatment with TG restored SOCE activation in preS2-LHBS cells as a result of the blockage of SERCA-dependent calcium entry into the ER. These results indicate that a persistent STIM1-Orail connection depends on the calcium concentration in the ER. Scale bar, $10 \mu \mathrm{m}$. 
calpain-dependent cyclin A proteolysis has been implicated in centrosome overduplication in HBVmediated hepatocarcinogenesis [26]. We measured the number of centrosomes in hepatocytes with or without LHBS. Compared with the control cells, centrosome overduplication (centrosome number $\geq 3$ ) was increased by $8.6-$ and 22.8-fold in WT-LHBS and preS2-LHBS cells, respectively (Figure 5A). The inhibition of ER stress, SOCE, and calpain largely suppressed centrosome overduplication in preS2-LHBS cells. Specifically, a bile acid derivative, tauroursodeoxycholic acid (TUDCA), was used as a chemical chaperone to reduce ER stress [26]. Kifunensine (KIF) was used to reduce ER stress by suppressing ER-associated protein degradation [27]. As expected, the STIM1-depleted cells also displayed reduced centrosome numbers (Figure 5B). Using $\gamma$-tubulin and chromatin staining, we found that preS2-LHBS cells displayed abnormal chromosome alignment and multiple spindle poles during mitotic progression (Figure 5C). To further explore the consequence of centrosome overduplication, we monitored mitotic progression using time-lapse imaging and found that approximately $10 \%$ of the WT-LHBS and $26 \%$ of the preS2-LHBS cells underwent aberrant multipolar division (Figure 5D and Supplementary Movies). Furthermore, the inhibition of ER stress, SOCE, and calpain, as well as treatment with calcium chelators, reduced aberrant multipolar division in preS2-LHBS cells (Figure 5E).

As a direct outcome of aberrant multipolar division is chromosome number instability, we continued to investigate changes in overall chromosome numbers in hepatocytes in the presence of preS2-LHBS. The parental line NeHepLxHT used in this study is diploid, and more than $95 \%$ of cells contain 46 chromosomes [21]. This chromosome stability was maintained in the established control line but was disrupted in cells carrying WT-LHBS and preS2-LHBS (Figure 6A). The average chromosome numbers in WT-LHBS and preS2-LHBS cells were 60 and 65 , respectively. Whereas the majority of WT-LHBS and preS2-LHBS cells had 51-70 chromosomes, cells carrying more than 100 chromosomes were detected only in preS2-LHBS cells (Figure 6A). Thus, preS2LHBS has a stronger impact on chromosome instability than WT-LHBS cells. Next, we investigated whether the inhibition of SOCE machinery may block preS2LHBS-induced chromosome instability. To this end, preS2-LHBS cells were treated with SOCE inhibitors for 7 and 14 days before harvesting for chromosome karyotyping. As expected, long-term inhibition of the SOCE machinery by 2 -APB, $\mathrm{Gd}^{3+}$, or $\mathrm{La}^{3+}$ significantly reduced chromosome instability in preS2-LHBS cells in two weeks (Figure 6B and Supplementary Figure S1). Apart from chromosome instability, we also detected a 3 -fold increase in the formation of micronuclei in preS2LHBS cells. Coordinately, micronucleation in preS2LHBS cells was decreased by long-term SOCE inhibition
(Figure 6C, 6D). Finally, we investigated whether SOCE is essential for preS2-LHBS-mediated tumorigenesis in immune-deficient mice. PreS2-LHBS cells were infected with recombinant lentiviruses that expressed control or STIM1 shRNA. Successful depletion of STIM1 upon lentivirus treatment was confirmed by western blotting (Figure 6E). Next, a total of $1 \times 10^{6}$ lentivirus-treated cells were injected subcutaneously into nude mice for the observation of xenograft tumorigenesis. Tumorigenesis in the control and shSTIM1 groups occurred in $69 \%$ $(11 / 16)$ and $13 \%(2 / 16)$ of mice, respectively. In addition, tumor volume was significantly reduced in the shSTIM1 group (Figure 6F). Together, these results implied that the activation of SOCE machinery contributed to centrosome overduplication, chromosome instability, and in vivo tumorigenesis in hepatocytes carrying preS2-LHBS (Figure 6G).

\section{DISCUSSION}

Since their initial discovery in the early 1970s, GGHs have been implicated as a diagnostic biomarker of chronic HBV infection and as a preneoplastic lesion for the development of $\mathrm{HCC}[6,25,28]$. Here we provide a mechanistic link between ER stress, SOCE, and chromosome instability in hepatocytes carrying preS2LHBS, a leading cause of type II GGHs. We show that ER stress increased the ER-PM connection through the STIM1Orail interaction, which subsequently initiated SOCE and thereby contributed to centrosome overduplication, multipolar division, and chromosome instability.

ER stress is a self-defense mechanism that is triggered by the presence of unfolded/malfolded proteins (also known as the unfolded protein response, UPR), the accumulation of these proteins (ER-overloaded response, EOR), or the disruption of calcium homeostasis in the ER [29, 30]. During chronic HBV infection, mutations occurred in the preS regions were found in GGHs with the accumulation of surface antigens, indicating that these proteins are trapped in the ER due to their inability to undergo proper folding [9]. PreS2-LHBS triggers both UPR and EOR, as implied from activations of UPR and EOR downstream signaling pathways $[6,14,26,31-33]$. As a secondary effect, the stress also leads to a sustained calcium leak from the ER and thereby increases the cytosolic calcium concentration [34]. This calcium effect is likely mediated by the calcium release channels ryanodine-receptor (RyR) or inositol 1,4,5-trisphosphate $\left(\mathrm{IP}_{3}\right)$-receptor $\left(\mathrm{IP}_{3} \mathrm{R}\right)$ or the newly identified translocon $[35,36]$. As a result, calcium is reduced in the ER, which can be rescued by increasing the calcium influx from extracellular resources, thereby replenishing ER calcium [37]. This mechanism is named store-operated calcium entry/SOCE because the calcium influx is triggered by calcium storage in the ER [38].

STIM1 is a calcium sensor in the ER. It has a single transmembrane domain with a calcium-binding EF-hand 
A
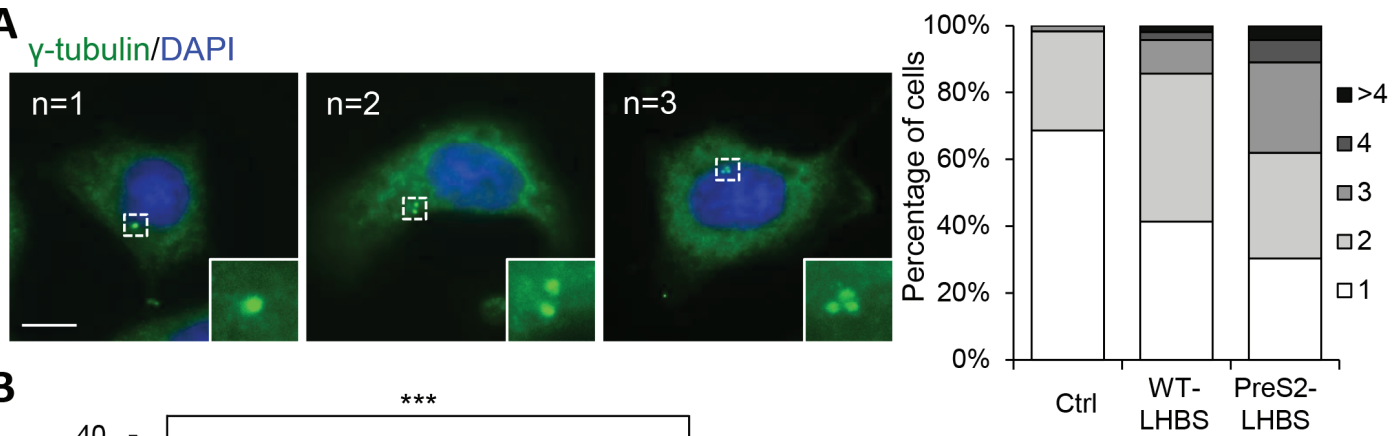

B

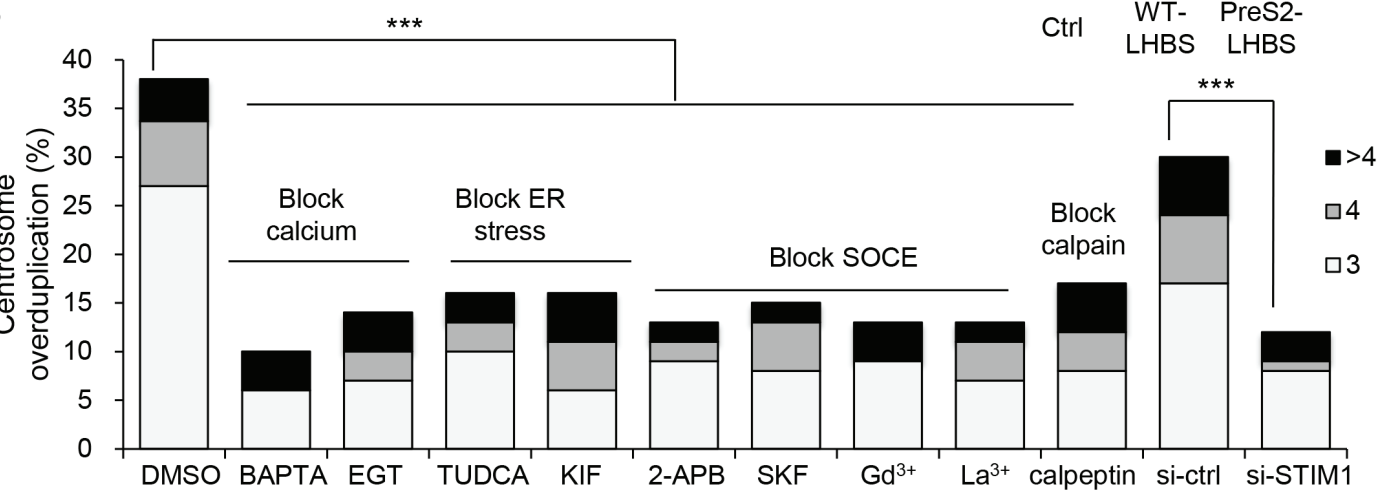

C y-tubulin/DAPI

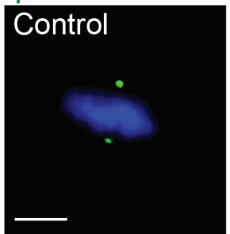

D A
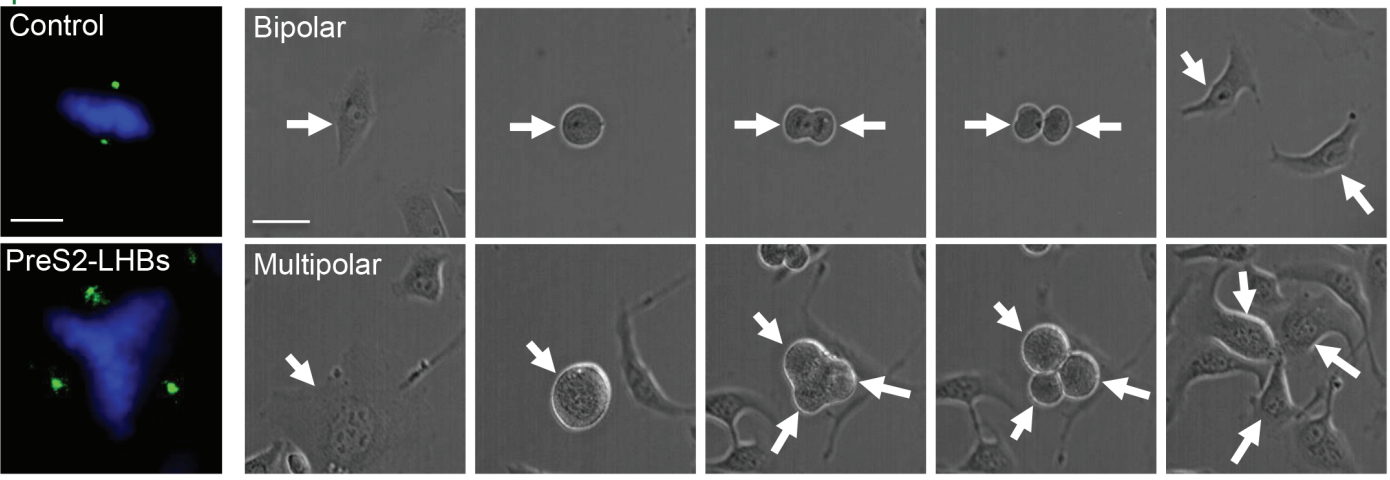

$\mathbf{E}$

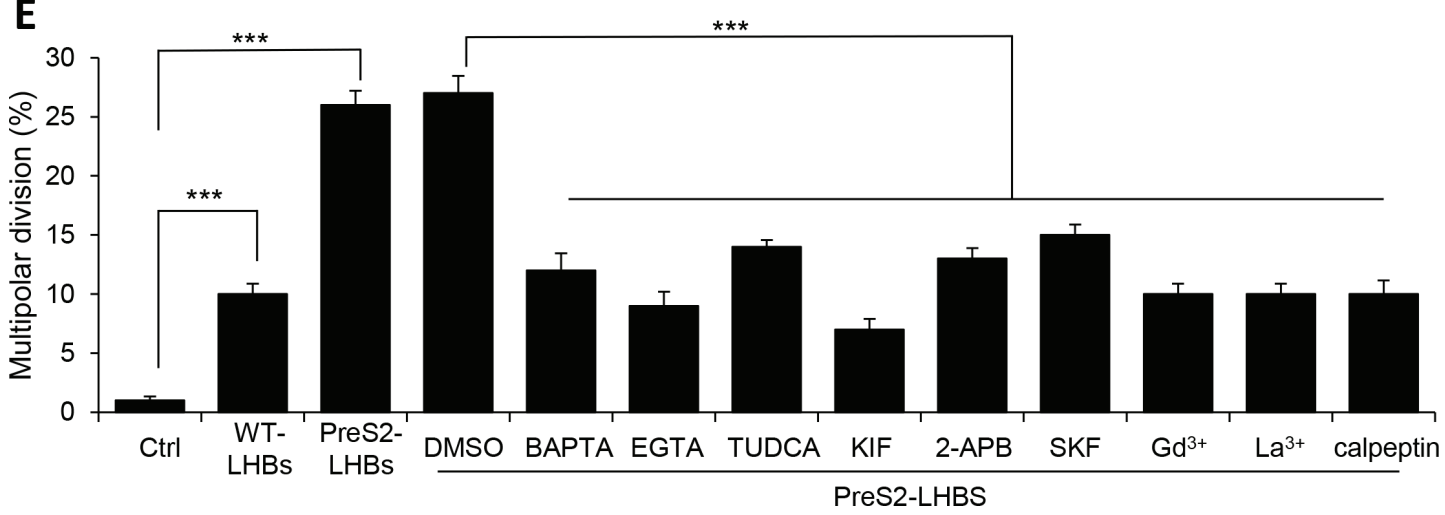

Figure 5: SOCE promotes centrosome overduplication and multipolar division. (A) Representative immunofluorescence images of cells carrying 1 to 3 centrosomes, as indicated by $\boldsymbol{\gamma}$-tubulin (green)-positive foci. Scale bar, $10 \mu \mathrm{m}$. Overall population of cells carrying different number of centrosomes in control, WT-LHBS, and preS2-LHBS cells is shown in the right panel. At least 200 cells were counted in each group, and the percentages of cells carrying different number of chromosomes are shown. (B) Centrosome overduplication in preS2LHBS cells was suppressed by calcium chelators (BAPTA-AM, EGTA) and inhibitors of ER stress (TUDCA, KIF), SOCE (2-APB, SKF96365, $\mathrm{Gd}^{3+}, \mathrm{La}^{3+}$ ), and calpain (calpeptin), as well as by the depletion of STIM1. Percentage of cells carrying 3 or more centrosomes following these treatments are shown. (C) Representative images showing cells that underwent bipolar (upper) or multipolar (lower) chromosome alignment during mitosis. Spindle poles and chromosomes are indicated by $\gamma$-tubulin (green) and DAPI (blue), respectively. Scale bar, $10 \mu \mathrm{m}$. (D) Time-lapse DIC images displaying cells undergoing bipolar and multipolar divisions. Arrows indicate either the mother or daughter cells as appropriate. Scale bar, $10 \mu \mathrm{m}$. (E) Percentages of cells that underwent multipolar division are shown as indicated. Multipolar division in preS2-LHBS cells was suppressed upon inhibition of ER stress, SOCE, or calpain. ${ }^{* * *} p<0.001$. 
A

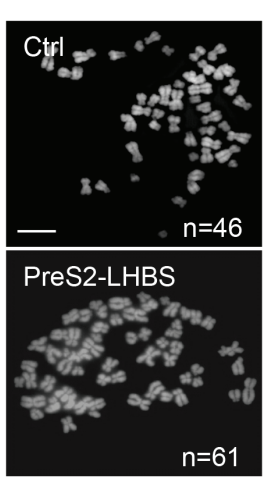

C

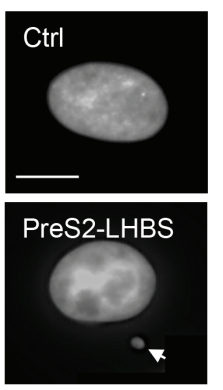

$\mathbf{G}$

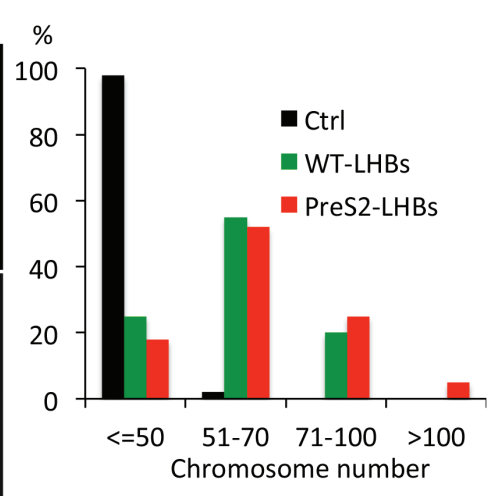

D

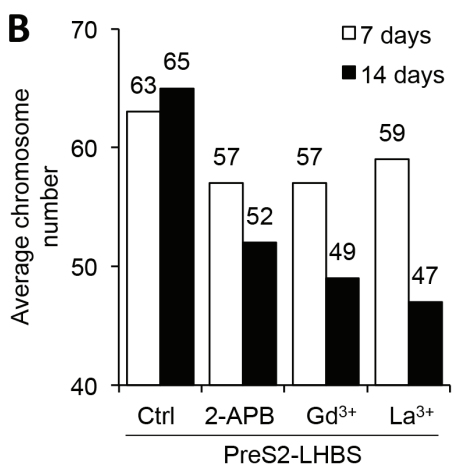

E

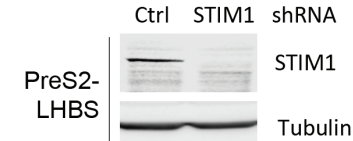

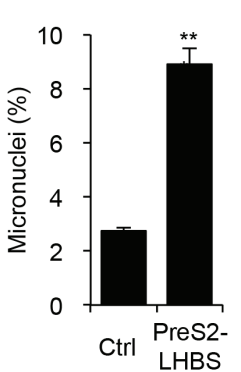

HBS

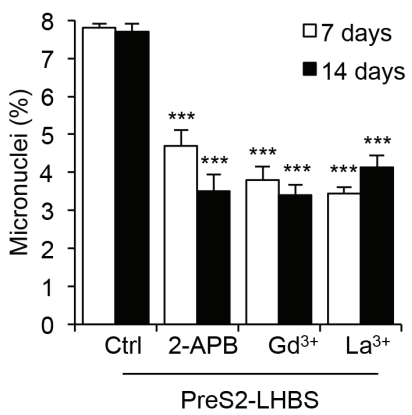

$\mathbf{F}$
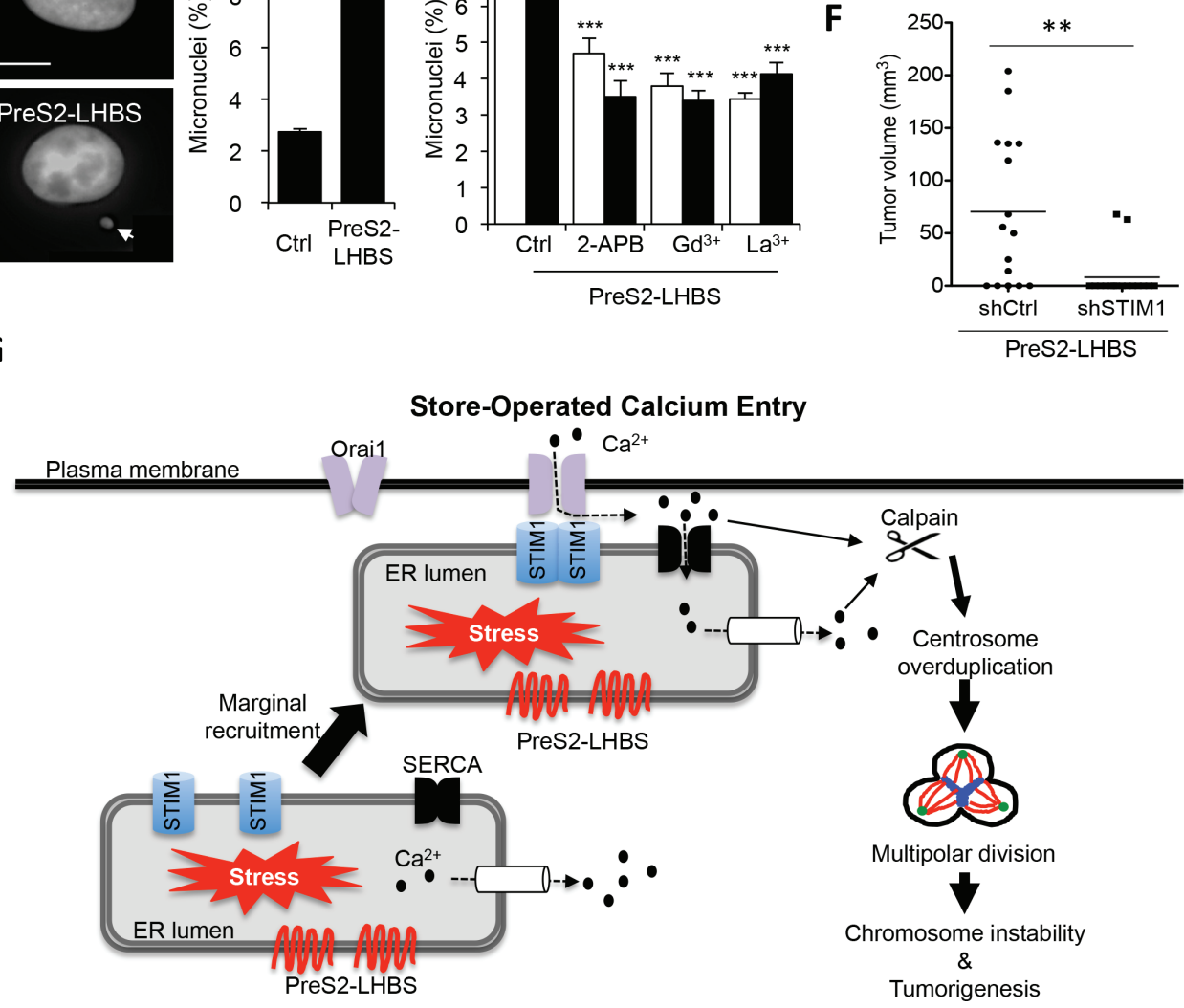

Figure 6: PreS2-LHBS promotes SOCE-dependent chromosome instability. (A) Overall chromosome numbers of control and LHBS-positive cells were examined by chromosome spreading. Representative chromosome spreads in control and preS2-LHBS cells are shown on the left. Scale bar, $10 \mu \mathrm{m}$. At least 200 spreads were counted per experiment. Quantitative chromosome number distribution is shown on the right. (B) The average chromosome number in preS2-LHBS cells was reduced following long-term SOCE inhibition by 2-APB, $\mathrm{Gd}^{3+}$, and $\mathrm{La}^{3+}$. At least 200 spreads were counted per experiment. Numbers shown on top of each column indicate the average chromosome numbers. (C) An increased number of cells that carried micronuclei (arrow-indicated) was detected in preS2-LHBS cells stained with DAPI. Scale bar, $10 \mu \mathrm{m}$. (D) The percentage of preS2-LHBS cells that contained micronuclei was reduced following longterm SOCE inhibition. $* * * p 0.001$. (E) Depletion of STIM1 protein expression in preS2-LHBS cells was confirmed upon treatment of recombinant lentiviruses carrying STIM1 shRNA. Tubulin expression was detected as an internal control. (F) STIM1 knockdown suppressed xenografted tumorigenesis of preS2-LHBS cells. PreS2-LHBS cells infected with recombinant lentiviruses for the expressions of control or STIM1 shRNA were injected subcutaneously into nude mice for the observation of in vivo tumorigenesis for 2 months. Tumor volumes are presented as scatter dot plot with mean values $(n=16) .{ }^{* *} p<0.01$. (G) Illustration of the cause and consequences of persistent ER-PM connections in type II GGHs. PreS2-LHBS triggers ER stress and promotes calcium release from the ER. Reduced ER calcium storage provokes STIM1 oligomerization and the recruitment of the STIM1-resident ER toward the PM-localized Orail. These actions initiate calcium influx, increase cytoplasmic calcium concentrations, and thereby provoke calpain activation and centrosome overduplication. Mitosis that proceeds with extra centrosomes results in abnormal multipolar division and ultimately produces chromosome instability. 
motif near the N-terminus, which is located in the ER lumen [39]. A decrease in ER luminal calcium concentration results in the dissociation of calcium from the EF-hand motif, which, in turn, leads to STIM1 oligomerization [40]. Accordingly, ER stress-induced calcium leak provoke STIM1 oligomerization. A recent model predicted that STIM1 dimers are involved in crosslinking Orail channels, with implications for the kinetics and localization of Orai1 channel opening [41]. Thus, the formation of STIM1-Orai1 clusters indicates not only the ER-PM junction sites but also where calcium enters the cell.

The present study implies that preS2-LHBS is recruited toward the plasma membrane, along with other ER resident proteins, upon SOCE activation. To support this notion, increased peripheral distribution of ER-resident GRP78 was detected in preS2-LHBS cells and in control lines after thapsigargin stimulation (Supplementary Figure S2). Based on the following reasons, we conclude that the marginal recruitment of preS2-LHBS depends on interactions between STIM1 and Orail but not LHBS. First, STIM1 depletion is sufficient to abolish the recruitment of preS2-LHBS to the cell margin (Figure 3C). Second, preS2-LHBS is not directly associated with STIM1 (Supplementary Figure S3). Third, increased ER-PM connection was detected upon SOCE activation in control cells even in the absence of LHBS (Figure 2A). These results imply that the marginal distribution of preS2-LHBS is a result of ER stress-mediated SOCE activation, not by the protein-protein interaction between preS2-LHBS and STIM1.

What is unique for the induction of SOCE during chronic HBV infection? Once the ER calcium concentration is replenished, SOCE should be inactivated, and the ER returns to the resting stage and dissociates from the PM. However, marginal distribution of preS2LHBS is constantly detected in type II GGHs (Figure 1A), indicating that the stress is never resolved in these hepatocytes. What keeps SOCE active in type II GGHs is the next question to be solved. We reasonably suspect that ER calcium influx may quickly leak out again in the presence of preS2-LHBS. To support this notion, preS2LHBS was treated with the high calcium condition in which the ER calcium leak is prevented by high cytosolic calcium concentration. In this condition, STIM1-Orai1 connected puncta were dissociated in preS2-LHBS cells, indicating that the persistent STIM1-Orail interaction depends on ER calcium storage (Figure 4). Furthermore, high calcium environments did not disrupt STIM1-Orai1 interactions when ER calcium replenishment was blocked by thapsigargin. These results imply that persistent ER stress, which constantly promotes the efflux of calcium from the ER, sustains SOCE activation in type II GGHs. As the stress conditions were not resolved, the interaction between STIM1 and Orai1 persisted in the cells.

PreS truncations are known as gain-of-function oncogenic mutations. PreS2-LHBS may promote hepatocarcinogenesis through ER stress-dependent and -independent pathways $[17,42]$. Notably, ER stress alone is insufficient to induce carcinogenesis because cell cycle progression may be attenuated by downstream signaling [30]. Alternatively, hepatocytes may cope with ER stress via an adaptive UPR, which includes the enhancement of protein folding and degradation in the ER [43]. This is likely the case for GGHs because ER chaperone proteins are increased in GGHs that carry preS1 and preS2 mutants in the liver [44]. In addition, preS2-LHBS may act as a gene transactivator to activate PKC/c-Raf-1/MAP2 kinase, $\mathrm{NF}-\kappa \mathrm{B}$, and mTOR signal cascades, as well as cyclin A and Bcl-2 upregulation, to facilitate cell proliferation $[14,45-48]$. The present study implies that type II GGHs are prone to aneuploidy if mitotic division occurs in the presence of extra centrosomes. Exactly how much genetic information was changed in type II GGHs is not clear. Notably, hepatocytes of various ploidy classes ( $4 \mathrm{n}$, $8 \mathrm{n}$, and $16 \mathrm{n}$ ) typically emerge during liver postnatal development [49]. It will be of interest to explore the influence of HBV on genomic integrity in the context of diverse hepatocyte polyploidy in the future.

Still, why it takes decades to develop HCC in patients with chronic HBV infection is unknown. We consider two possible explanations. First, during the early stages of HBV infection, hepatocytes expressing viral antigens are obviously eliminated by host immunity, and therefore, the expansion of virus-infected hepatocytes is likely limited. This immune pressure pushes the emergence of preS mutants, as a result of immune escape. This is supported by the increasing prevalence of type II GGHs and preS2 mutants in advanced stages of chronic HBV infection [6]. Second, HCC is characterized with complex genomic composition [20, 50, 51], implying that the development of $\mathrm{HCC}$ requires the accumulation of multiple genetic changes during disease progression. Whereas the development of preS2 mutants facilitates genetic defects, endogenous tumor suppressor genes can still limit aberrant cell cycle progression of virus-infected hepatocytes until their functionalities are compromised by further genetic changes. Thus, the risk of HCC was increased by time in chronic $\mathrm{HBV}$ carriers [52]. If the effect of host immunity is removed, the presence of a preS2 mutant alone should speed up the disease progression. In fact, the presence of preS mutants is a known risk of liver cirrhosis and HCC [53]. In addition, preS2 mutants were detected in nearly half of all children with HCC [11]. Accordingly, whether and when immuneescape and oncogenic preS2 mutants occur in the liver may set the clock for advanced disease progression in patients with chronic HBV infection.

Apart from preS2-LHBS, another viral oncoproduct, $\mathrm{HBx}$, was shown to affect calcium signaling in hepatocytes through a completely different mechanism. $\mathrm{HBx}$ interacts directly with $\mathrm{Bcl}-2$ and $\mathrm{Bcl}-\mathrm{xL}$ through its Bcl-2-homology-3-like motif, and this interaction 
not only elevates cytosolic calcium but also provokes cell death and viral replication [54]. The inhibition of mitochondrial calcium channels blocked HBX-mediated HBV replication, indicating that cytosolic calcium is regulated by limited mitochondrial calcium uptake [55]. Unlike preS2-LHBS, which can be abundantly detected in type II GGHs, the expression of HBx is often limited in $\mathrm{HCC}$ and a small population of surrounding parenchyma cells [56]. In cirrhotic livers, HBx was detected in small subpopulations of type II GGHs [57]. The co-expression of HBx and preS2-LHBS displayed a synergistic effect on several oncogenic signals, such as vascular endothelial growth factor-A, Akt, and mTOR, as well as xenograft tumorigenesis [57]. Whether and how $\mathrm{HBx}$ coordinates with preS2-LHBS in the development of HBV-related $\mathrm{HCC}$ requires further investigation.

In conclusion, the activation of SOCE machinery explains the marginal recruitment of preS2-LHBS and the subsequent chromosome instability. To the best of our knowledge, this study provides the first mechanistic link between calcium homeostasis and chromosome instability in the pathogenesis of HBV. Notably, SOCE was shown to be essential for the migration, invasion, and proliferation of hepatoma cells and cervical carcinoma [58, 59]. Together, these studies highlight SOCE as a promising therapeutic target to control disease progression during chronic HBV infection.

\section{MATERIALS AND METHODS}

\section{Cell culture, treatments, and bacmam transduction}

The immortalized hepatic progenitor cell line NeHepLxHT [21] was purchased from and cultured as suggested by American Type Culture Collection (ATCC, Manassas, VA, USA). To generate stable cell lines, NeHepLxHT cells were infected with recombinant lentiviruses that carried SNAP (as a control) or SNAPtagged WT and preS2 mutant LHBS genes (deletion nucleotides 2-55) and selected by $2 \mu \mathrm{g} / \mathrm{ml}$ puromycin. Viable cells were transiently labeled with cell-permeable SNAP-505 dye (New England Biolabs, MA, USA) and subjected to cell sorting. The following reagents were used: TG (thapsigargin, $2 \mu \mathrm{M}$, Sigma-Aldrich, St Louis, MO, USA), KIF (kifunensine, $50 \mu \mathrm{M}$, SigmaAldrich), TUDCA (tauroursodeoxycholic acid [sodium salt], $100 \mu \mathrm{M}$, Sigma-Aldrich), EGTA (ethylene glycol tetraacetic acid, $100 \mu \mathrm{M}$, Biokit, Miaoli, Taiwan), BAPTAAM (4 $\mu \mathrm{M}$, Calbiochem, San Diego, CA, USA), and calpeptin $(20 \mu \mathrm{M}$, Calbiochem). The SOCE inhibitors (2-APB, SKF96365, $\mathrm{Gd}^{3+}$, and $\mathrm{La}^{3+}$ ) and siRNA targeting STIM1 were used as previously described [60]. STIM1 and Orail subcellular localization was monitored via transient transfection of cells with plasmids that encoded mOrangeOrail and EGFP-STIM1 for 48 hr. To monitor ER and
PM localization, the cells were treated with BacMam baculovirus (CellLight ER-GFP and PM-RFP, Molecular Probes, Eugene, OR, USA) for $48 \mathrm{hr}$ prior to fixation.

\section{Immunohistochemical and double immunofluorescence staining}

For hepatic LHBS staining, paraffin-embedded tissues were pre-warmed at $65^{\circ} \mathrm{C}$ for $15 \mathrm{~min}$ and treated with xylene for paraffin removal. Antigen retrieval was performed by boiling the samples in a tissue pretreatment buffer solution (Invitrogen) for $30 \mathrm{~min}$. The slides were then incubated with mouse anti-preS1 for $1 \mathrm{hr}$ and assessed using the EnVision+ System-HRP kit (DAKO, Carpintena, CA, USA). For double immunofluorescence staining, the cells were fixed and stained with the indicated primary antibodies for $1 \mathrm{hr}$, followed by secondary antibodies conjugated with Alexa-488, Alexa-594, or Alexa-647 (Molecular Probes). The cells were mounted with mounting medium that contained DAPI. The images were acquired with a Zeiss LSM780 confocal microscope or an automated Leica DMI6000 inverted microscope equipped with an HCX PL FL 20x/NA0.4 objective and an EMCCD camera (Andor Luca R, Belfast, UK) as indicated. The following primary antibodies were used in this study: mouse anti-preS1 (a gift from Ningshao Xia, Xiamen University, China), rabbit anti-SNAP tag (P9310S, New England Biolabs, MA, USA), rabbit anti-STIM1 (PA523623, Thermo Scientific, IL, USA), and mouse anti- $\gamma$ tubulin (sc-17787, Santa Cruz, Dallas, Texas, USA).

\section{Monitoring mitotic progression by time-lapse live cell microscopy}

For live cell imaging, individual cells were isolated and cultured in 35-mm Petri dishes (Ibidi, Germany) for a minimum of $12 \mathrm{hr}$ prior to imaging. The cells were maintained on a microscope stage incubator at $37^{\circ} \mathrm{C}$ with a humidified atmosphere and $5 \% \mathrm{CO}_{2}$ throughout the experiment. Multi-positional time-lapse imaging was performed using an automated Leica DMI6000 inverted microscope equipped with an HCX PL FL 20x/NA0.4 objective and an Andor Luca R EMCCD camera. To monitor mitotic cell progression, sequential differential interference contrast (DIC) images of the cells were obtained at 10-min intervals for $24 \mathrm{hr}$ and analyzed using MetaMorph software. At least 100 cells were monitored per experiment, and the experiment was independently repeated three times.

\section{Measuring intracellular calcium and SOCE activity}

The total cellular calcium concentration was measured by loading cells with Fura-2/acetoxymethyl ester (Fura-2/AM, Molecular Probes) at $37^{\circ} \mathrm{C}$ for $30 \mathrm{~min}$. The 
cells were washed three times with phosphate-buffered saline (PBS) and imaged. To monitor calcium influx and SOCE activity, the cells were loaded with Fura-2/AM at $37^{\circ} \mathrm{C}$ for $30 \mathrm{~min}$ and subsequently incubated in a calciumfree medium that contained $2 \mu \mathrm{M}$ thapsigargin for $30 \mathrm{~min}$ to deplete intracellular calcium stores. Calcium influx was measured after the addition of $2 \mathrm{mM}$ calcium to the culture medium. The cells were imaged using an Olympus IX71 inverted microscope equipped with a xenon illumination system, an IMAGO CCD camera, and a Polychrome IV monochromator to maintain the excitation wavelength between 340 and $380 \mathrm{~nm}$ (TILL Photonics, Grafelfing, Germany). The cytosolic calcium concentration, total amount of calcium efflux from the ER (ER-releasable $\mathrm{Ca}^{2+}$ ), and calcium influx from the SOCE machinery were calculated using Origin 6.0 software (OriginLab, Northampton, MA, USA).

\section{Metaphase chromosome spread}

The cells were treated with demecolcine $(50 \mathrm{ng} / \mathrm{ml}$, Sigma) overnight; the mitotic cells were subsequently collected by mitotic shake-off. The cells were resuspended in $75 \mathrm{mM} \mathrm{KCl}$ and incubated at room temperature for $20 \mathrm{~min}$. The cells were then resuspended in freshly prepared Carnoy's solution (ice-cold methanol:glacial acetic acid $=3: 1$ ) for $30 \mathrm{~min}$ on ice. For chromosome spread analysis, the mitotic cells in Carnoy's solution were dropped onto glass slides, air-dried, and stained with DAPI. Images were acquired using a Leica DMI6000 inverted microscope.

\section{Lentivirus production and infection}

Lentiviruses encoding STIM1 or control luciferase small-hairpin RNAs (shRNA) were obtained from the TRC lentiviral shRNA library in the National RNAi Core Facility of Academia Sinica, Taiwan. The targeting sequences of specific shRNAs are as follows: STIM1-1 (TRCN-0000149588: 5'- CGATGA GATCAACCTTGCTAA-3'); STIM1-2 (TRCN-0000358 717: 5'- TGGTGGTGTCTATCGTTATTG-3'); STIM1-3 (TRCN-0000180131: 5'- CCACTCACAGTGGTTCTGT TT-3'); and luciferase (TRCN000072246: 5'- CAAAT CACAGAATCGTCGTAT-3'). Lentivirus production was performed according to the guidelines of the National RNAi Core Facility of Academia Sinica. To efficiently deplete endogenous STIM1, a mixture of STIM1-1, STIM-2, and STIM-3 lentiviruses was used.

\section{Xenograft tumorigenesis}

For tumor xenograft experiments, BALB/c nu/ nu nude mice (6-8 weeks old) were purchased from National Laboratory Animal Center, Taiwan and housed in microisolator cages at a specific pathogen-free facility. PreS2-LHBS cells were infected with recombinant lentiviruses expressing control or STIM1 shRNA for $48 \mathrm{~h}$ before being trypsinized, washed, and resuspended in PBS. A total of $1 \times 10^{6}$ cells in $100 \mu \mathrm{l}$ (PBS: matrigel $=1: 1)$ were subcutaneously injected into the nude mice. Tumor volumes and numbers were measured at 2 months after injection. The expression of preS2-LHBS in tumor nodules was further confirmed by Western blotting.

\section{ACKNOWLEDGMENTS AND FUNDING}

We appreciate Prof. Szecheng J. Lo for his insightful comments and suggestions. We also thank Prof. Ningshao Xia (Xiamen University, China) for sharing the mouse anti-preS1 antibody. This work is supported by Ministry of Science and Technology in Taiwan (NSC-101-2320-B007-006-MY3 and MOST-104-2320-B-007-003 to Dr. LH Wang; MOST 104-2320-B-218 -002 -MY3 to Dr. IJ Su), National Tsing Hua University (102N2052E1 to Dr. LHC Wang), and the Center of Infectious Diseases and Signal Transduction, National Cheng Kung University, Taiwan.

\section{CONFLICTS OF INTEREST}

The authors declare no conflicts of interest.

\section{REFERENCES}

1. Chen DS. From hepatitis to hepatoma: lessons from type B viral hepatitis. Science. 1993; 262:369-370.

2. Chisari FV, Ferrari C. Hepatitis B virus immunopathogenesis. Annu Rev Immunol. 1995; 13:29-60.

3. Beasley RP. Hepatitis B virus. The major etiology of hepatocellular carcinoma. Cancer. 1988; 61:1942-1956.

4. Arzumanyan A, Reis HM, Feitelson MA. Pathogenic mechanisms in HBV- and HCV-associated hepatocellular carcinoma. Nat Rev Cancer. 2013; 13:123-135.

5. Su IJ, Lai MY, Hsu HC, Chen DS, Yang PM, Chuang SM, Sung JL. Diverse virological, histopathological and prognostic implications of seroconversion from hepatitis B e antigen to anti-HBe in chronic hepatitis B virus infection. J Hepatol. 1986; 3:182-189.

6. Su IJ, Wang HC, Wu HC, Huang WY. Ground glass hepatocytes contain pre-S mutants and represent preneoplastic lesions in chronic hepatitis B virus infection. J Gastroenterol Hepatol. 2008; 23:1169-1174.

7. Fan YF, Lu CC, Chen WC, Yao WJ, Wang HC, Chang TT, Lei HY, Shiau AL, Su IJ. Prevalence and significance of hepatitis B virus (HBV) pre-S mutants in serum and liver at different replicative stages of chronic HBV infection. Hepatology. 2001; 33:277-286.

8. Chen BF, Liu CJ, Jow GM, Chen PJ, Kao JH, Chen DS. High prevalence and mapping of pre-S deletion in hepatitis B virus carriers with progressive liver diseases. Gastroenterology. 2006; 130:1153-1168. 
9. Wang HC, Wu HC, Chen CF, Fausto N, Lei HY, Su IJ. Different types of ground glass hepatocytes in chronic hepatitis B virus infection contain specific pre-S mutants that may induce endoplasmic reticulum stress. Am J Pathol. 2003; 163:2441-2449.

10. Fang ZL, Sabin CA, Dong BQ, Wei SC, Chen QY, Fang KX, Yang JY, Huang J, Wang XY, Harrison TJ. Hepatitis B virus pre-S deletion mutations are a risk factor for hepatocellular carcinoma: a matched nested case-control study. J Gen Virol. 2008; 89:2882-2890.

11. Huang HP, Hsu HY, Chen CL, Ni YH, Wang HY, Tsuei DJ, Chiang CL, Tsai YC, Chen HL, Chang MH. Pre-S2 deletions of hepatitis B virus and hepatocellular carcinoma in children. Pediatr Res. 2010; 67:90-94.

12. Abe K, Thung SN, Wu HC, Tran TT, Le Hoang $\mathrm{P}$, Truong KD, Inui A, Jang JJ, Su IJ. Pre-S2 deletion mutants of hepatitis B virus could have an important role in hepatocarcinogenesis in Asian children. Cancer Sci. 2009; 100:2249-2254.

13. Fan YF, Lu CC, Chang YC, Chang TT, Lin PW, Lei HY, $\mathrm{Su}$ IJ. Identification of a pre-S2 mutant in hepatocytes expressing a novel marginal pattern of surface antigen in advanced diseases of chronic hepatitis B virus infection. J Gastroenterol Hepatol. 2000; 15:519-528.

14. Wang HC, Chang WT, Chang WW, Wu HC, Huang W, Lei HY, Lai MD, Fausto N, Su IJ. Hepatitis B virus pre-S2 mutant upregulates cyclin A expression and induces nodular proliferation of hepatocytes. Hepatology. 2005; 41:761-770.

15. Wang HC, Huang W, Lai MD, Su IJ. Hepatitis B virus pre-S mutants, endoplasmic reticulum stress and hepatocarcinogenesis. Cancer Sci. 2006; 97:683-688.

16. Wang LH, Huang W, Lai MD, Su IJ. Aberrant cyclin A expression and centrosome overduplication induced by hepatitis B virus pre-S2 mutants and its implication in hepatocarcinogenesis. Carcinogenesis. 2012; 33:466-472.

17. Su IJ, Wang LH, Hsieh WC, Wu HC, Teng CF, Tsai HW, Huang W. The emerging role of hepatitis B virus pre-S2 deletion mutant proteins in HBV tumorigenesis. J Biomed Sci. 2014; 21:98.

18. Hsieh YH, Su IJ, Wang HC, Tsai JH, Huang YJ, Chang WW, Lai MD, Lei HY, Huang W. Hepatitis B virus pre-S2 mutant surface antigen induces degradation of cyclin-dependent kinase inhibitor p27Kip1 through c-Jun activation domainbinding protein 1. Mol Cancer Res. 2007; 5:1063-1072.

19. Hsieh YH, Chang YY, Su IJ, Yen CJ, Liu YR, Liu RJ, Hsieh WC, Tsai HW, Wang LH, Huang W. Hepatitis B virus pre-S2 mutant large surface protein inhibits DNA double-strand break repair and leads to genome instability in hepatocarcinogenesis. J Pathol. 2015; 236:337-347.

20. Roessler S, Long EL, Budhu A, Chen Y, Zhao X, Ji J, Walker R, Jia HL, Ye QH, Qin LX, Tang ZY, He P, Hunter $\mathrm{KW}$, et al. Integrative genomic identification of genes on $8 \mathrm{p}$ associated with hepatocellular carcinoma progression and patient survival. Gastroenterology. 2012; 142:957-966 e912.
21. Reid Y, Gaddipati JP, Yadav D, Kantor J. Establishment of a human neonatal hepatocyte cell line. In Vitro Cell Dev Biol Anim. 2009; 45:535-542.

22. Parekh AB, Putney JW, Jr. Store-operated calcium channels. Physiol Rev. 2005; 85:757-810.

23. Berna-Erro A, Woodard GE, Rosado JA. Orais and STIMs: physiological mechanisms and disease. J Cell Mol Med. 2012; 16:407-424.

24. Mathai AM, Alexander J, Kuo FY, Torbenson M, Swanson PE, Yeh MM. Type II ground-glass hepatocytes as a marker of hepatocellular carcinoma in chronic hepatitis B. Human pathology. 2013.

25. Popper $\mathrm{H}$. The ground glass hepatocyte as a diagnostic hint. Hum Pathol. 1975; 6:517-520.

26. Ozcan U, Yilmaz E, Ozcan L, Furuhashi M, Vaillancourt E, Smith RO, Gorgun CZ, Hotamisligil GS. Chemical chaperones reduce ER stress and restore glucose homeostasis in a mouse model of type 2 diabetes. Science. 2006; 313:1137-1140.

27. Wang F, Song W, Brancati G, Segatori L. Inhibition of endoplasmic reticulum-associated degradation rescues native folding in loss of function protein misfolding diseases. J Biol Chem. 2011; 286:43454-43464.

28. Hadziyannis S, Gerber MA, Vissoulis C, Popper H. Cytoplasmic hepatitis B antigen in "ground-glass" hepatocytes of carriers. Arch Pathol. 1973; 96:327-330.

29. Malhi H, Kaufman RJ. Endoplasmic reticulum stress in liver disease. J Hepatol. 2011; 54:795-809.

30. Kaufman RJ. Orchestrating the unfolded protein response in health and disease. J Clin Invest. 2002; 110:1389-1398.

31. Hung JH, Su IJ, Lei HY, Wang HC, Lin WC, Chang WT, Huang W, Chang WC, Chang YS, Chen CC, Lai MD. Endoplasmic reticulum stress stimulates the expression of cyclooxygenase-2 through activation of NF-kappaB and pp38 mitogen-activated protein kinase. J Biol Chem. 2004; 279:46384-46392.

32. Hsieh YH, Su IJ, Wang HC, Chang WW, Lei HY, Lai MD, Chang WT, Huang W. Pre-S mutant surface antigens in chronic hepatitis B virus infection induce oxidative stress and DNA damage. Carcinogenesis. 2004; 25:2023-2032.

33. Yang JC, Teng CF, Wu HC, Tsai HW, Chuang HC, Tsai TF, Hsu YH, Huang W, Wu LW, Su IJ. Enhanced expression of vascular endothelial growth factor-A in ground glass hepatocytes and its implication in hepatitis B virus hepatocarcinogenesis. Hepatology. 2009; 49:1962-1971.

34. Deniaud A, Sharaf el dein O, Maillier E, Poncet D, Kroemer G, Lemaire C, Brenner C. Endoplasmic reticulum stress induces calcium-dependent permeability transition, mitochondrial outer membrane permeabilization and apoptosis. Oncogene. 2008; 27:285-299.

35. Hammadi M, Oulidi A, Gackiere F, Katsogiannou M, Slomianny C, Roudbaraki M, Dewailly E, Delcourt P, Lepage G, Lotteau S, Ducreux S, Prevarskaya N, Van Coppenolle F. Modulation of ER stress and apoptosis 
by endoplasmic reticulum calcium leak via translocon during unfolded protein response: involvement of GRP78. Faseb J. 2013; 27:1600-1609.

36. Mekahli D, Bultynck G, Parys JB, De Smedt H, Missiaen L. Endoplasmic-reticulum calcium depletion and disease. Cold Spring Harb Perspect Biol. 2011; 3.

37. Garcia-Sancho J. The coupling of plasma membrane calcium entry to calcium uptake by endoplasmic reticulum and mitochondria. The Journal of physiology. 2014; 592:261-268.

38. Berna-Erro A, Redondo PC, Rosado JA. Store-operated $\mathrm{Ca}(2+)$ entry. Adv Exp Med Biol. 2012; 740:349-382.

39. Park CY, Hoover PJ, Mullins FM, Bachhawat P, Covington ED, Raunser S, Walz T, Garcia KC, Dolmetsch RE, Lewis RS. STIM1 clusters and activates CRAC channels via direct binding of a cytosolic domain to Orai1. Cell. 2009; 136:876-890.

40. Luik RM, Wang B, Prakriya M, Wu MM, Lewis RS. Oligomerization of STIM1 couples ER calcium depletion to CRAC channel activation. Nature. 2008; 454:538-542.

41. Zhou Y, Wang $\mathrm{X}$, Wang $\mathrm{X}$, Loktionova NA, Cai X, Nwokonko RM, Vrana E, Wang Y, Rothberg BS, Gill DL. STIM1 dimers undergo unimolecular coupling to activate Orail channels. Nat Commun. 2015; 6:8395.

42. Pollicino T, Cacciola I, Saffioti F, Raimondo G. Hepatitis B virus PreS/S gene variants: pathobiology and clinical implications. J Hepatol. 2014; 61:408-417.

43. Ji C, Kaplowitz N. ER stress: can the liver cope? J Hepatol. 2006; 45:321-333.

44. Wang HC, Wu HC, Chen CF, Fausto N, Lei HY, $\mathrm{Su}$ IJ. Different types of ground glass hepatocytes in chronic hepatitis B virus infection contain specific pre-S mutants that may induce endoplasmic reticulum stress. The American journal of pathology. 2003; 163:2441-2449.

45. Hung JH, Teng YN, Wang LH, Su IJ, Wang CC, Huang W, Lee KH, Lu KY, Wang LH. Induction of Bcl-2 expression by hepatitis $\mathrm{B}$ virus pre-S2 mutant large surface protein resistance to 5-fluorouracil treatment in Huh-7 cells. PLoS One. 2011; 6:e28977.

46. Hildt E, Munz B, Saher G, Reifenberg K, Hofschneider PH. The PreS2 activator MHBs(t) of hepatitis B virus activates c-raf-1/Erk2 signaling in transgenic mice. EMBO J. 2002; 21:525-535.

47. Hildt E, Hofschneider PH. The PreS2 activators of the hepatitis B virus: activators of tumour promoter pathways. Recent Results Cancer Res. 1998; 154:315-329.

48. Teng CF, Wu HC, Tsai HW, Shiah HS, Huang W, Su IJ. Novel feedback inhibition of surface antigen synthesis by mammalian target of rapamycin (mTOR) signal and its implication for hepatitis B virus tumorigenesis and therapy. Hepatology. 2011; 54:1199-1207.
49. Celton-Morizur S, Desdouets C. Polyploidization of liver cells. Adv Exp Med Biol. 2010; 676:123-135.

50. Yeh SH, Chen PJ, Lai MY, Chen DS. Allelic loss on chromosomes $4 \mathrm{q}$ and $16 \mathrm{q}$ in hepatocellular carcinoma: association with elevated alpha-fetoprotein production. Gastroenterology. 1996; 110:184-192.

51. Homayounfar K, Schwarz A, Enders C, Cameron S, Baumhoer D, Ramadori G, Lorf T, Gunawan B, Sander B. Etiologic influence on chromosomal aberrations in European hepatocellular carcinoma identified by CGH. Pathology, research and practice. 2013; 209:380-387.

52. Lin CL, Kao JH. Risk stratification for hepatitis B virus related hepatocellular carcinoma. J Gastroenterol Hepatol. $2013 ; 28: 10-17$.

53. Chen $\mathrm{CH}$, Hung $\mathrm{CH}$, Lee $\mathrm{CM}, \mathrm{Hu} \mathrm{TH}$, Wang JH, Wang JC, $\mathrm{Lu}$ SN, Changchien CS. Pre-S deletion and complex mutations of hepatitis B virus related to advanced liver disease in $\mathrm{HBeAg}$-negative patients. Gastroenterology. 2007; 133:1466-1474.

54. Geng X, Huang C, Qin Y, McCombs JE, Yuan Q, Harry BL, Palmer AE, Xia NS, Xue D. Hepatitis B virus X protein targets $\mathrm{Bcl}-2$ proteins to increase intracellular calcium, required for virus replication and cell death induction. Proc Natl Acad Sci U S A. 2012; 109:18471-18476.

55. Bouchard MJ, Wang LH, Schneider RJ. Calcium signaling by $\mathrm{HBx}$ protein in hepatitis B virus DNA replication. Science. 2001; 294:2376-2378.

56. Su Q, Schroder CH, Hofmann WJ, Otto G, Pichlmayr R, Bannasch P. Expression of hepatitis B virus X protein in $\mathrm{HBV}$-infected human livers and hepatocellular carcinomas. Hepatology. 1998; 27:1109-1120.

57. Wu HC, Tsai HW, Teng CF, Hsieh WC, Lin YJ, Wang LH, Yuan Q, Su IJ. Ground-glass hepatocytes co-expressing hepatitis $\mathrm{B}$ virus $\mathrm{X}$ protein and surface antigens exhibit enhanced oncogenic effects and tumorigenesis. Hum Pathol. 2014; 45:1294-1301.

58. Yang N, Tang Y, Wang F, Zhang H, Xu D, Shen Y, Sun S, Yang G. Blockade of store-operated $\mathrm{Ca}(2+)$ entry inhibits hepatocarcinoma cell migration and invasion by regulating focal adhesion turnover. Cancer Lett. 2013; 330:163-169.

59. Chen YF, Chiu WT, Chen YT, Lin PY, Huang HJ, Chou CY, Chang HC, Tang MJ, Shen MR. Calcium store sensor stromal-interaction molecule 1-dependent signaling plays an important role in cervical cancer growth, migration, and angiogenesis. Proc Natl Acad Sci U S A. 2011; 108:15225-15230. 